\title{
Electric current of an electrified jet issuing from a long metallic tube
}

\author{
F. J. HIG UER A ${ }^{\dagger}$ \\ E. T. S. Ingenieros Aeronáuticos, UPM, Pza Cardenal Cisneros 3, 28040 Madrid, Spain
}

\begin{abstract}
This paper presents an analysis of the transport of electric current in a jet of an electrically conducting liquid discharging from a metallic tube into a gas or a vacuum, and subject to an electric field due to a high voltage applied between the tube and a far electrode. The flow, the surface charge and the electric field are computed in the current transfer region of the jet, where conduction current in the liquid becomes surface current due to the convection of electric charge accumulated at its surface. The electric current computed as a function of the flow rate of the liquid injected through the tube increases first as the square root of this flow rate, levels to a nearly constant value when the flow rate is increased and finally sets to a linear increase when the flow rate is further increased. The current increases linearly with the applied voltage at small and moderate values of this variable, and faster than linearly at high voltages. The characteristic length and structure of the current transfer region are determined. Order-of-magnitude estimates for jets which are only weakly stretched by the electric stresses are worked out that qualitatively account for some of the numerical results.
\end{abstract}

Key words: capillary flows, electrohydrodynamic effects

\section{Introduction}

The techniques of electrospray and electrospinning are well suited to produce nearly monodisperse drops and fibres of electrically conducting liquids with controllable diameters in the range from tens of nanometres to tens of micrometres (Barrero \& Loscertales 2007; Fernández de la Mora 2007). Typically, the liquid to be processed is injected through a metallic capillary tube into a gas or a vacuum and forms a meniscus at the end of the tube. An electric field due to a high voltage applied between the tube and another electrode induces a conduction current in the liquid which accumulates electric charge at its surface and leads to electric stresses that elongate the meniscus in the direction of the field. In the cone-jet regime (Cloupeau \& Prunet-Foch 1989), the meniscus becomes a Taylor cone (Taylor 1964) with a thin jet issuing from its tip. The jet carries an electric current which is made of the conduction current in the liquid and a surface current due to the convection of the electric charge accumulated at its surface. The conduction contribution to the current dominates in the meniscus and the convection contribution dominates in the jet, with a current transfer region in between where the two contributions are of the same order and the current is transferred to the surface. Fernández de la Mora \& Loscertales (1994) 
found that, when the conductivity of the liquid is high and the flow rate injected through the tube is not much larger than the minimum for which a cone-jet is possible (Cloupeau \& Prunet-Foch 1989), the electric current depends only on the flow rate and the physical properties of the liquid, and it increases as the square root of the flow rate. Under these conditions, the radius of the jet and the size of the current transfer region are small compared to the size of the meniscus and any other dimension of the system, and the electric field around the current transfer region is that of a Taylor cone independently of the configuration of the electrodes. The square root law is then obtained under many different conditions of the flow, and it has become the hallmark of the cone-jet regime; see Fernández de la Mora (2007) for a review.

This universality is lost, and the electric current departs from the square root law and becomes apparatus-dependent, when the current transfer region ceases to be small, either because it becomes long compared to the size of the conical meniscus (Higuera 2004) or because the radius of the jet ceases to be small compared to the radius of the tube, so that no conical meniscus exists. The latter condition is typical of the high flow rates often used in electrospinning. Hartman et al. (1999) carried out experiments and numerical computations with ethylene glycol and $n$ butanol for various shapes of the injection nozzle and found that the exponent of the power law fitting their measured current for high flow rates is smaller than $1 / 2$, and that the current depends on the shape of the electrode. Hohman et al. (2001), in experiments with glycerol and polyethylene oxide (PEO)/water solutions in a parallel plate electrode configuration in which the injection tube protrudes from one of the plates, found that the electric current increases roughly linearly with the flow rate. The current also increases linearly with the voltage applied between the electrodes, except for very high voltages, for which the increase is faster than linear. In additional experiments with the same apparatus, Shin et al. (2001) observed that the slope of the current/flow rate characteristic of their high-conductivity $\mathrm{PEO} /$ water solutions changes when the jet becomes unstable to asymmetric (whipping) perturbations.

More recently, Gundabala, Vilanova \& Fernández-Nieves (2010) set up an electrospray in a microfluidic device where a stream of very viscous conducting oil is surrounded by a co-flow of dielectric oil. They found that the current depends weakly on the flow rate and increases linearly with the applied voltage. That this current is not due to conduction in the liquid but to convection of the surface charge by the electric-shear-induced surface velocity was proved by an ingenious combination of experimental measurements and qualitative analysis. Riboux et al. (2011) carried out experiments with glycerine in a bath of hexane using a needle-plate electrode configuration. They report a current that increases as the power $1 / 5$ of the flow rate and the power $4 / 3$ of the voltage, which they attribute to differences between the charge transport mechanisms in their jets and the jets of regular electrosprays in air (Fernández de la Mora 2007). Contrary to the viscous outer oil in the experiments of Gundabala et al. (2010), the relatively inviscid hexane has little effect on the current transfer region in the experiments of Riboux et al., though the bath still affects the evolution of the whipping jet further downstream. Larriba \& Fernández de la Mora (2010) obtained similar results for the current/flow rate and current/voltage characteristics of a cone-jet of an ionic liquid in a bath of heptane, and explained them as effects of the space charge due to the drops of the electrospray.

In this paper, the transport of current in an electrified jet issuing from a long metallic tube is investigated numerically using a quasi-unidirectional approximation for the flow in the jet. The numerical results show that the current/flow rate characteristic 
evolves from nearly square root at small flow rates to linear at very large flow rates, with a plateau of nearly constant current in between. The computed current increases with the applied voltage, almost linearly at small and moderate voltages, and faster than linearly at high voltages. Order-of-magnitude estimates for the transfer region of weakly stretched jets are worked out that qualitatively account for some features of the solution at high flow rates and moderate voltages. The numerical results are in qualitative agreement with experimental results reviewed above. However, the idealized electrode geometry assumed here, together with the possibility of long-range electrical interactions due to the charge of the jet far from tube (see $\$ 3.2$ ), prevents more quantitative comparisons.

\section{Formulation}

A flow rate $Q$ of a liquid of density $\rho$, viscosity $\mu$, surface tension $\gamma$, electrical conductivity $K$ and electrical permittivity $\epsilon_{0} \epsilon$ is injected through a metallic capillary tube of inner diameter $a$ into a dielectric medium of permittivity $\epsilon_{0}$ and negligible density and viscosity (a gas or a vacuum). The outer surface of the tube is a paraboloid of revolution of mean curvature at the tip $a^{-1}$ and truncated at a distance $a / 2$ from this tip. In this configuration, a high voltage applied between the tube and a far electrode leads to the electric field $\boldsymbol{E}_{\infty}=-\nabla \varphi_{\infty}$ with $\varphi_{\infty}=-A \ln \left\{\left[\sqrt{x^{2}+r^{2}}+x\right] / a\right\}$ for $\sqrt{x^{2}+r^{2}} \gg a$; see, e.g. Landau \& Lifshitz (1960). Here $x$ and $r$ are distances along and normal to the axis of the tube, measured from its end, and $A$ is a constant proportional to the applied voltage ( $A$ is equal to the applied voltage divided by $\ln (2 L / a)$ when the far electrode is a paraboloid confocal with the needle at a distance $L \gg a$ from it).

This electric field is modified by the presence of the liquid. Assuming that the flow is axisymmetric, with a free surface $r=r_{s}(x)$ where the density of free surface charge is $\sigma(x)$ (both to be found), the electric potentials outside and inside the liquid, $\varphi_{1}$ and $\varphi_{2}$ respectively, with $\boldsymbol{E}_{i}=-\nabla \varphi_{i},(i=1,2)$, satisfy Laplace's equation in each medium:

$$
\nabla^{2} \varphi_{1}=0 \text { outside the liquid and } \nabla^{2} \varphi_{2}=0 \text { inside the liquid, }
$$

with the boundary conditions

$$
\epsilon_{0}\left(E_{1 n}-\epsilon E_{2 n}\right)=\sigma \quad \text { and } \quad E_{1 t}=E_{2 t} \quad \text { at } r=r_{s}(x)
$$

(see, e.g. Landau \& Lifshitz 1960); $\varphi_{i}=0$ at the surface of the metallic tube and $\varphi_{i} \sim \varphi_{\infty}$ far from the tube. Here $E_{i n}$ and $E_{i t}$ are the components of the electric field $\boldsymbol{E}_{i}$ normal to the surface (pointing away from the liquid) and tangent to it. The presence of an electric field and free electric charge at the surface of the liquid leads to electric stresses on the surface, whose components normal and tangent to the surface are (Landau \& Lifshitz 1960; Saville 1997)

$$
\tau_{n}^{e}=\frac{\epsilon_{0}}{2}\left(E_{1 n}^{2}-\epsilon E_{2 n}^{2}\right)+\frac{\epsilon_{0}}{2}(\epsilon-1) E_{1 t}^{2} \quad \text { and } \quad \tau_{t}^{e}=\sigma E_{1 t} .
$$

The velocity and pressure in the liquid obey the mass and momentum conservation equations. In what follows, the flow is assumed to be stationary and these equations are approximated using a quasi-unidirectional model valid for $\left|\mathrm{d} r_{s} / \mathrm{d} x\right| \ll 1$, which is justified in the jet and marginally valid in the meniscus; see, e.g. Feng (2002). Accounting for the electric and surface tension stresses at the surface of the liquid, and assuming a plug flow velocity profile $v(x)$, mass and momentum balances for an 
element of the jet give

$$
\begin{gathered}
\pi r_{s}^{2} v=Q, \\
\frac{\partial}{\partial x}\left(\pi r_{s}^{2} \rho v^{2}\right)=\frac{\partial}{\partial x}\left(3 \pi r_{s}^{2} \mu \frac{\partial v}{\partial x}\right)+\pi r_{s}^{2} \frac{\partial}{\partial x}\left(\tau_{n}^{e}-\gamma \mathscr{C}\right)+2 \pi r_{s} \tau_{t}^{e},
\end{gathered}
$$

where $\mathscr{C} \approx 1 / r_{s}$ is twice the mean curvature of the surface. These equations are to be solved with the boundary condition $r_{s}(0)=a$ (or $v(0)=Q / \pi a^{2}$ ) at the end of the tube, where the surface of the liquid is assumed to be pinned, and the condition that the jet evolves smoothly towards the asymptotic state (3.5) far downstream; see $\$ 3.2$ below.

The density of surface charge obeys the transport equation

$$
\frac{\mathrm{d} I_{s}}{\mathrm{~d} x}=2 \pi r_{s} K E_{2 n}
$$

where $I_{s}=2 \pi r_{s} \sigma v$ is the surface current due to the convection of the surface charge by the flow and the right-hand side of (2.6) is the rate of change of $I_{s}$ along the jet due to the component of the conduction current density normal to the surface. Equation (2.6) will be solved with the condition $\sigma(0)=0\left(\right.$ or $\left.I_{5}(0)=0\right)$, which expresses that the interface generated by the discharge of the liquid from the tube has no initial charge. Numerical tests show that this condition has little effect on the current for realistic values of the parameters of the problem.

The mathematical problem formulated in this section can be written in a dimensionless form scaling distances with the inner radius of the tube $a$, velocities with the viscous-capillary velocity $v_{c}=\gamma / \mu$, electric fields with $E_{c}=\left(\gamma / \epsilon_{0} a\right)^{1 / 2}$, and the electric potentials and electric charge density with $E_{c} a$ and $\epsilon_{0} E_{c}$. The dimensionless problem depends on the five parameters

$$
C a=\frac{\mu Q}{\gamma a^{2}}, \quad V_{\infty}=\frac{\epsilon_{0}^{1 / 2} A}{\gamma^{1 / 2} a^{1 / 2}}, \quad \epsilon, \quad \Lambda=\frac{\mu K a}{\epsilon_{0} \gamma}, \quad R e=\frac{\rho \gamma a}{\mu^{2}},
$$

and can be obtained from the dimensional formulation above by setting $a=\epsilon_{0}=\gamma=\mu=1$ and replacing $Q, A$ (in the expression of $\varphi_{\infty}$ ), $K$ and $\rho$ by their dimensionless counterparts $C a, V_{\infty}, \Lambda$ and $R e$, respectively. Here $\Lambda / \epsilon$ is the ratio of the hydrodynamic time $a / v_{c}$ to the electric relaxation time $t_{e}=\epsilon_{0} \epsilon / K$.

For the numerical treatment, a boundary element method is used to solve the Laplace's equations (2.1) with their boundary conditions, while the non-stationary forms of (2.4)-(2.6) (with terms $\partial\left(\pi r_{s}^{2} \rho v\right) / \partial t$ and $\partial\left(2 \pi r_{s} \sigma\right) / \partial t$ added to the left-hand sides of (2.5) and (2.6), and (2.4) replaced by $\left.\partial\left(\pi r_{s}^{2}\right) / \partial t+\partial\left(\pi r_{s}^{2} v\right) / \partial x=0\right)$ are marched in time until the solution converges to a stationary state. As in previous computations (Higuera 2010), the jet is truncated at a certain distance from the tube, $x_{00}$, chosen through numerical tests to ensure that the truncation has no important effect on the results; see also $\$ 3.2$.

\section{Results and discussion}

\subsection{Numerical results}

The electric current carried by the jet is $I=I_{s}+I_{b}$, which is a constant independent of $x$. Here $I_{s}=2 \pi r_{s} \sigma v$ is the convection current, which increases with $x$, and $I_{b}=2 \pi K \int_{0}^{r_{s}} E_{2 x} r \mathrm{~d} r$ is the conduction current, which decreases with $x$. There is a cross-over point, $x=x_{c}$, where $I_{s}\left(x_{c}\right)=I_{b}\left(x_{c}\right)$. The magnitudes $I$ and $x_{c}$, scaled with 

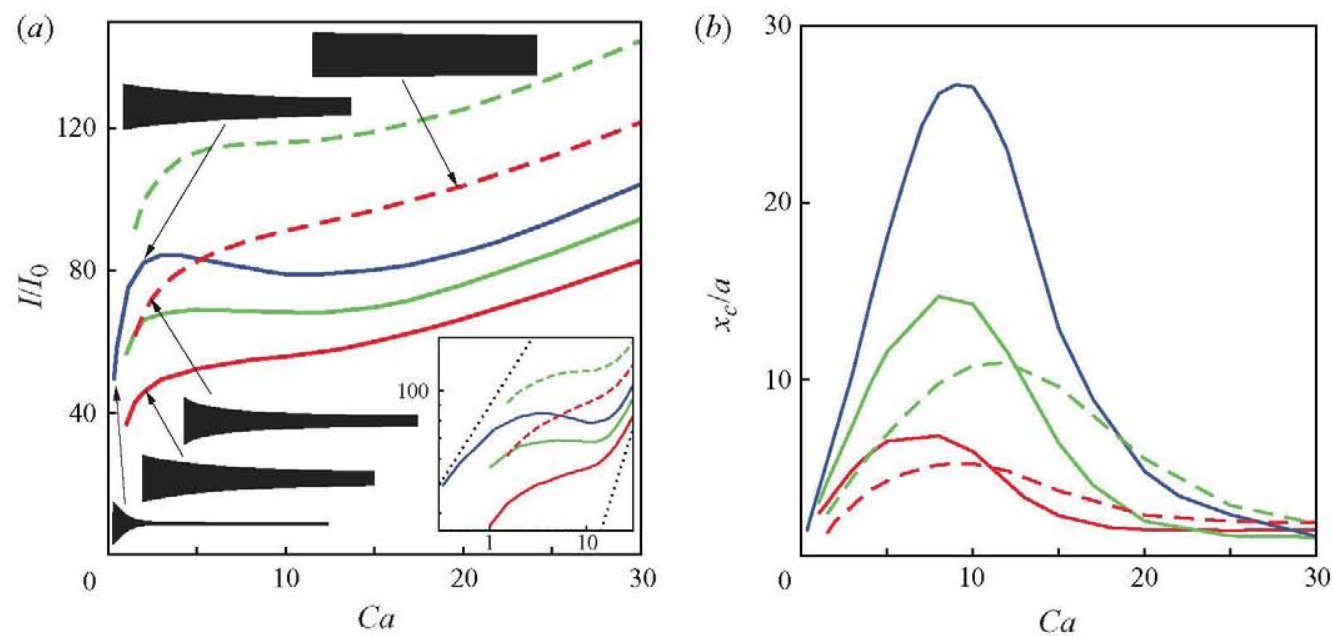

FIGURE 1. (Colour online available at journals.cambridge.org/FLM) Dimensionless electric current $(a)$ and dimensionless distance to the current cross-over point $(b)$ as functions of $\mathrm{Ca}$ for $\epsilon=20, R e=0.2, V_{\infty}=3$ (solid curves) and 4 (dashed curves), and $\Lambda=200$ (red), 500 (green) and 1000 (blue), increasing from bottom to top. (Results for $\Lambda=1000$ are shown only when $V_{\infty}=3$.) The dotted lines on the left- and right-hand sides of the logarithmic inset in $(a)$ have slopes $1 / 2$ and 1, respectively. Some contours of the liquid surface are shown to illustrate the variation of the stretching of the jet due to the electric force with $C a, V_{\infty}$ and $\Lambda$. The contours are, from bottom to top, for $\left(\mathrm{Ca}, V_{\infty}, \Lambda\right)=(0.3,3,1000),(3,3,200),(3,4,200),(3,3,1000)$ and $(20,4,200)$.

$I_{0}=\epsilon_{0}^{1 / 2} \gamma^{3 / 2} a^{1 / 2} / \mu$ and $a$, respectively, are shown in figure 1 as functions of the dimensionless flow rate $\mathrm{Ca}$ for constant values of other parameters. These values are similar to the values in the experiments of Riboux et al. (2011), except for $R e$, which is larger than in the experiments in order to ensure that the inertia of the liquid counts in the current transfer region around $x_{c}$ where the dominant contribution to the current changes from conduction to convection. The effect of decreasing $R e$ is discussed in $\$ 3.2$ below. As can be seen in figure 1, the electric current increases nearly as the square root of $\mathrm{Ca}$ for small values of this variable, for which the meniscus is conical with a thin jet (lowest contour in figure 1a) and the current transfer region is short compared to the radius of the tube. The computations cannot be extended to very small capillary numbers because the value of the dimensionless applied voltage $V_{\infty}$ is not suitable to attain the equilibrium of surface tension and normal electric stresses characteristic of a conical hydrostatic meniscus at very small $\mathrm{Ca}$ (Taylor 1964).

When $\mathrm{Ca}$ increases, the cross-over point $x_{c}$ shifts streamwise and the transfer of current to the surface of the liquid occurs away from the tube, in a region where the electric field due to the applied voltage is $A / x$. The increase of the current with $\mathrm{Ca}$ then becomes moderate; the current seems to level to a constant value, as in the experiments of Hohman et al. (2001) and Riboux et al. (2011), and even decreases slightly with increasing $\mathrm{Ca}$ (upper solid curves in figure $1 a$ ). At the same time, the radius of the jet increases and $r_{s}\left(x_{c}\right) / a$ ceases to be small. When $C a$ is further increased, the cross-over point recedes towards the tube, the radius of the jet approaches the radius of the tube and the current tends to increase linearly with $\mathrm{Ca}$.

Figure 2 shows the density of surface charge and the conduction and convection contributions to the current as functions of the streamwise distance along the jet. For 

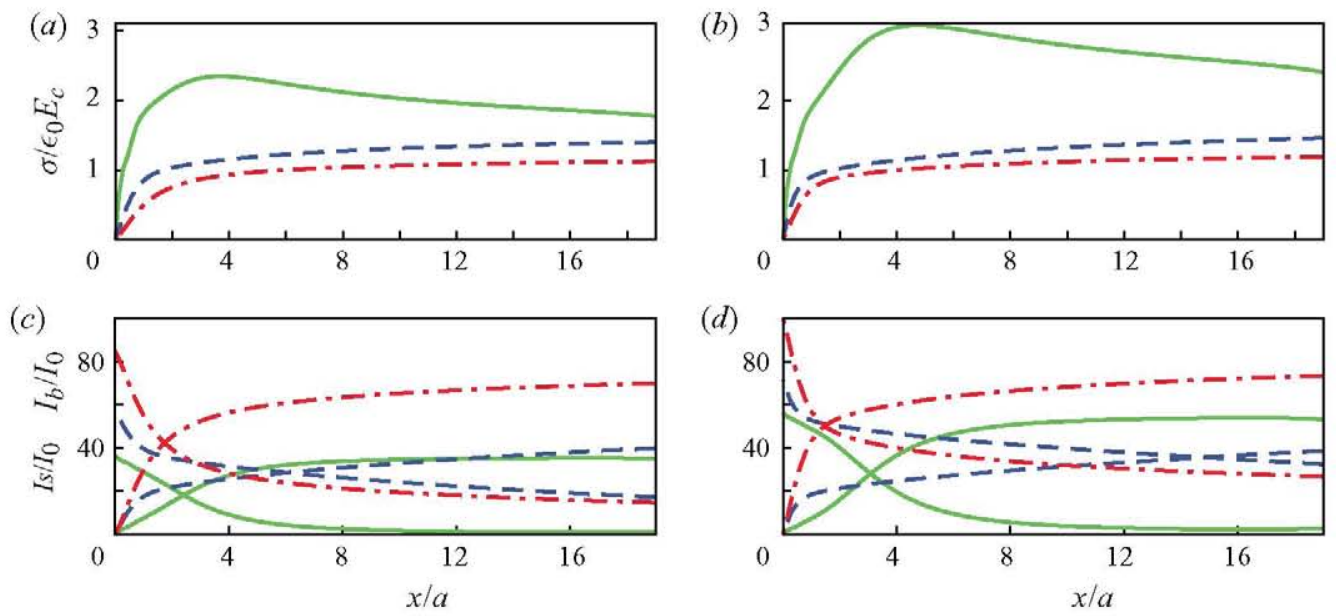

Figure 2. (Colour online) Dimensionless density of surface charge (upper row) and dimensionless convection (increasing) and conduction (decreasing) contributions to the current as functions of the dimensionless streamwise distance for $\epsilon=20, \operatorname{Re}=0.2, V_{\infty}=3, \Lambda=200$ $((a)$ and $(c))$ and $500((b)$ and $(d))$, and $C a=1$ (solid, green), 10 (dashed, blue) and 30 (dash-dotted, red).

the smallest value of $\mathrm{Ca}$ shown (solid curves), the evolution of the charge density and the current is smooth in the current transfer region and resembles the evolution of these variables in a cone-jet; see, e.g. Higuera (2003). As $\mathrm{Ca}$ increases, the evolution splits into two stages. There is a relatively fast variation in a leading relaxation region whose length increases with $C a$ and decreases with $\Lambda$, followed by a slower variation further downstream (where the density of surface charge reaches a shallow maximum which is not visible in figures $2 a$ and $2 b$ ). The second variation becomes small compared to the first when $\mathrm{Ca}$ increases further, leading to a nearly uniform $\sigma$ that depends weakly on $C a$ and increases with $V_{\infty}$ (result not displayed), and to a convection current that increases with $C a$ and $V_{\infty}$. At high $C a$, the convection current is large compared to the conduction current remaining in the jet at the end of the relaxation region, so that the subsequent transfer of this current to the surface due to the smooth decrease of the axial field with streamwise distance causes only a small additional increase of the convection current.

The electric current and the distance from the tube to the cross-over point are shown in figure 3 as functions of $V_{\infty}$. The electric current increases linearly with $V_{\infty}$ for small and moderate values of this parameter, for which the radius of the jet is not much smaller than the radius of the tube, and it increases somewhat faster than linearly for large values of $V_{\infty}$, for which the decrease of the radius of the jet due to the electric shear stress in (2.5) is somewhat more marked. In both regimes, the current depends weakly on the dimensionless conductivity $\Lambda$, which confirms that it is not due to conduction in the liquid. The distance $x_{c}$ to the cross-over point does increase with $\Lambda$. In addition, it is a decreasing function of $C a$ at small $V_{\infty}$ and an increasing function of $\mathrm{Ca}$ at large $V_{\infty}$, for which the cross-over point recedes when $V_{\infty}$ increases. The numerical computations cease to converge to a stationary state when $V_{\infty}$ decreases below a certain minimum that increases when $C a$ decreases. This result probably reflects the instability of the weakly electrified jet to axisymmetric perturbations captured by the quasi-unidirectional model. 

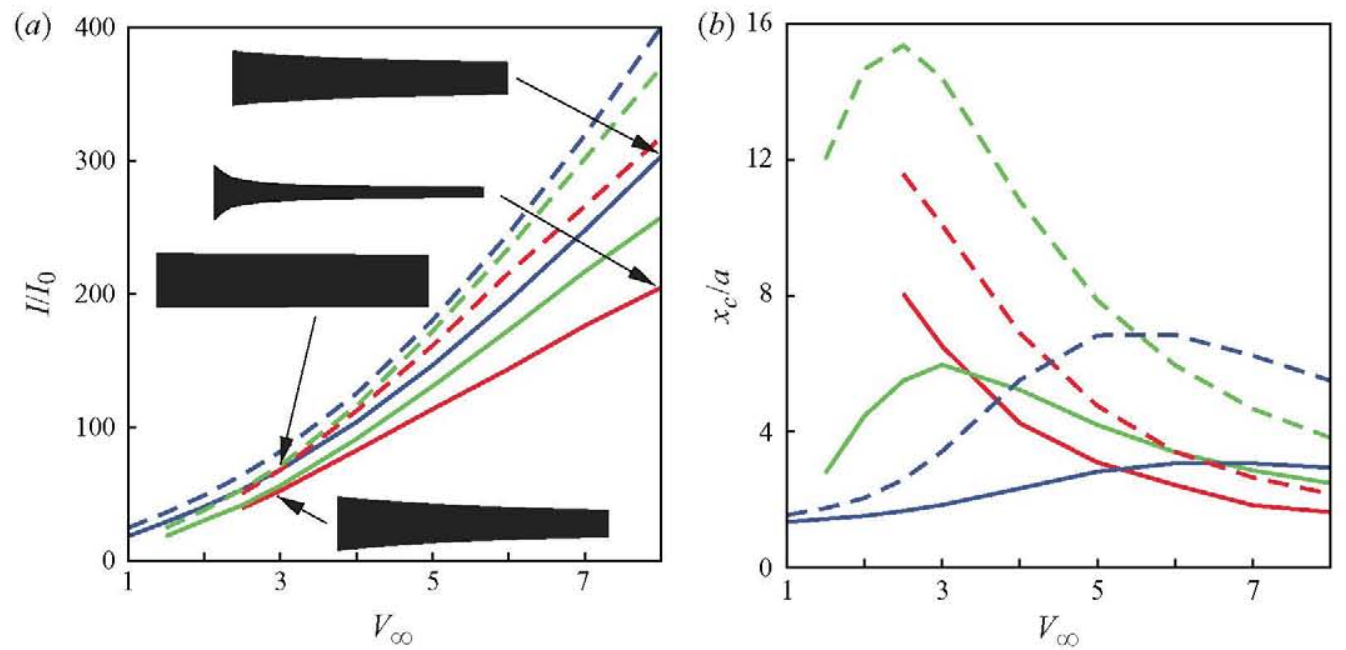

Figure 3. (Colour online) Dimensionless electric current $(a)$ and dimensionless distance to the current cross-over point $(b)$ as functions of $V_{\infty}$ for $\epsilon=20, R e=0.2, \Lambda=200$ (solid curves) and 500 (dashed curves), and $C a=5$ (red), 10 (green) and 20 (blue), increasing from bottom to top (on the right-hand side in $(b)$ ). The contours shown in $(a)$ are, from bottom to top, for $\left(C a, V_{\infty}, \Lambda\right)=(5,3,200),(20,3,200),(5,8,200)$ and $(20,8,200)$.

\subsection{Order-of-magnitude estimations}

Some of the numerical results presented above can be accounted for using simple order-of-magnitude estimations. These are similar to well-known estimations for the cone-jet regime, where conduction current becomes convection current in a small region immersed in the electric field of a Taylor cone (Fernández de la Mora \& Loscertales 1994; Gañán-Calvo, Dávila \& Barrero 1997; Higuera 2003). Only the modifications of these results for the case when the applied field in the current transfer region is of the form $A / x$ will be discussed here.

Let $x_{c t}$ and $r_{s_{c t}}$ be the orders of the length of the current transfer region where $I_{s} \sim I_{b}$ and the radius of the jet in this region. Insofar as $x_{c t} \gg r_{s c t}$, the jet acts electrically as a line of charge which induces an electric field whose radial and axial components at the surface satisfy (see, e.g. Ashley \& Landahl 1965)

$$
E_{x}=\frac{\mathrm{d}\left(E_{r} r_{s}\right)}{\mathrm{d} x}\left[\ln \frac{r_{s}}{x_{c t}}+O(1)\right] .
$$

Here $E_{r} \approx \sigma / \epsilon_{0}$ past the relaxation region where the surface charge rises to its equilibrium value. The axial field induced by the charge of the jet must partially balance the applied field $A / x$ in order to prevent this field from entering the liquid for any $x \ll x_{c t}$, where it would lead to a conduction current larger than the total current of the jet. Leaving out a logarithmic factor, this condition gives $\sigma \sim \epsilon_{0} A / r_{s_{c t}}$.

The convection and conduction contributions to the current are $I_{s} \sim r_{s} \sigma v \sim \epsilon_{0} A Q / r_{s_{c t}}^{2}$ and $I_{b} \sim K r_{s_{c t}}^{2} A / x_{c t}$, respectively, in the current transfer region, where $v \sim Q / r_{s_{c t}}^{2}$ and the applied field finally enters the liquid. The condition $I_{s} \sim I_{b}$ gives

$$
\epsilon_{0} Q x_{c t} \sim K r_{s_{c t}}^{4},
$$

which is valid for any applied field; see Higuera (2010). 
The electric shear stress acting on the current transfer region of the jet is $\tau_{t}^{e}=\sigma E_{1 t} \sim \epsilon_{0} A^{2} /\left(r_{s_{c t}} x_{c t}\right)$. The axial electric force (last term of (2.5)) is balanced by the inertia of the liquid (left-hand side of (2.5)) for all but very small values of $R e$. Assuming that the axial electric force can noticeably stretch the jet, the balance of these terms reads $\rho Q^{2} /\left(r_{s_{c t}}^{2} x_{c t}\right) \sim \epsilon_{0} A^{2} / x_{c t}$, which determines $r_{s_{c t}}$. The length of the current transfer region can then be obtained from (3.2) and the order of the electric current from the expressions of $I_{s}$ or $I_{b}$ in the preceding paragraph. The results are

$$
\left.\begin{array}{c}
r_{s_{c t}} \sim \frac{\rho^{1 / 2} Q}{\epsilon_{0}^{1 / 2} A}=a \frac{R e^{1 / 2} C a}{V_{\infty}}, \quad x_{c t} \sim \frac{\rho^{2} K Q^{3}}{\epsilon_{0}^{3} A^{4}}=a \frac{R e^{2} \Lambda C a^{3}}{V_{\infty}^{4}}, \\
I \sim \frac{\epsilon_{0}^{2} A^{3}}{\rho Q}=I_{0} \frac{V_{\infty}^{3}}{\operatorname{ReCa}},
\end{array}\right\}
$$

where $I_{0}=\epsilon_{0}^{1 / 2} \gamma^{3 / 2} a^{1 / 2} / \mu$, as before. These estimates have been derived elsewhere (Higuera 2004). Their conditions of validity include (i) $\operatorname{Re}^{2} \Lambda C a^{3} / V_{\infty}^{4} \gg 1$, in order for the length of the current transfer region to be large compared to the radius of the tube, and (ii) $R e^{1 / 2} \mathrm{Ca} / V_{\infty} \ll 1$, in order for the radius of the jet to be small compared to the radius of the tube. The two conditions taken together require $V_{\infty}^{4 / 3} / R e^{2 / 3} \Lambda^{1 / 3} \ll C a \ll V_{\infty} / R e^{1 / 2}$. The lower bound of this range of $C a$ is not much smaller than the upper bound in the conditions of the computations of $\$ 3.1$ and the experiments of Riboux et al. (2011), so that the estimates (3.3) are only marginally applicable in these cases. In particular, the prediction that the electric current decreases as the inverse of the flow rate is not realized, though it is hinted at by the results of figure $1(a)$ for $V_{\infty}=3$ and $\Lambda=500$ and 1000 .

The applied electric field ceases to be of the form $A / x$ when condition (i) is not satisfied. If $\operatorname{Re}^{2} \Lambda C a^{3} / V_{\infty}^{4} \ll 1$ and the electric forces allow a hydrostatic meniscus (i.e. if $V_{\infty}=O(1)$ ), then the transfer of current occurs under the action of the electric field of a Taylor cone and the well-known $I \propto Q^{1 / 2}$ law is recovered. If condition (ii) is not satisfied, then the electric shear stress is not able to noticeably decrease the radius of the jet. This leads to assume $r_{s_{c t}} \sim a$ to replace the balance of forces above (3.3). Carrying this new condition to (3.2) and the expressions of the current gives the modified estimates

$$
r_{s_{c t}} \sim a, \quad x_{c t} \sim \frac{K a^{4}}{\epsilon_{0} Q}=a \frac{A}{C a}, \quad I \sim \frac{\epsilon_{0} A Q}{a^{2}}=I_{0} C a V_{\infty} .
$$

An electric current increasing linearly with the flow rate and the applied voltage is realized on the right-hand side of figure $1(a)$ and on the left-hand side of figure $3(a)$, where the radius of the jet is nearly equal to the radius of the tube and $\operatorname{Re}^{1 / 2} \mathrm{Ca} / V_{\infty}$ is moderately large. The decrease of $x_{c}$ with increasing $\mathrm{Ca}$ on the right-hand side of figure $1(b)$ also agrees with the prediction (3.4) for $x_{c t}$, while the increase of $x_{c}$ on the left-hand side of this figure is in qualitative agreement with (3.3). However, the inverse dependence of the electric current on $\mathrm{Ca}$ and its independence from $\Lambda$ predicted by (3.3) are at variance with the results of figure $3(a)$ for high $V_{\infty}$.

The computed radius of the jet is never much smaller than the radius of the tube, contrary to the assumption implicit in (3.3) (which therefore gives dimensional results independent of $a$ ). However, the axial electric force can still cause a moderate stretching of the jet which is not accounted for in (3.4). The effect of this stretching can be easily understood. Since the charge per unit length of the jet is nearly independent of $r_{s_{c t}}\left(2 \pi r_{s} \sigma \sim \epsilon_{0} A\right.$ according to the estimations at the beginning of this section), the radius of the jet affects the convection current $I_{s}=2 \pi r_{s} \sigma v$ mainly 
through the factor $v=Q / \pi r_{s}^{2}$. The balance of inertia and electric force, rewritten as $\Delta\left(r_{s}^{2} \rho v^{2}\right) / x_{c t} \sim \epsilon_{0} A^{2} / x_{c t}$ with $\Delta\left(r_{s}^{2} \rho v^{2}\right) \sim-\left(\rho Q^{2} / a^{2}\right)\left(\Delta r_{s} / a\right)$, gives the variation of the radius of the jet relative to the radius of the tube as $\Delta r_{s} / a \sim-V_{\infty}^{2} / R e C a^{2}$. This means that $r_{s c t}$ decreases when $C a$ decreases or $V_{\infty}$ increases. Therefore the electric current decreases less than linearly when $C a$ is decreased (as in figure $1 a$ ) and increases faster than linearly when $V_{\infty}$ is increased (as in figure $3 a$ ), provided $\Delta r_{s} / a$ is not very small, i.e. for small or moderate values of $C a$ and large values of $V_{\infty}$.

Additional computations show that the radius of the jet decreases, the electric current increases and the linear growth of the current with the capillary number is postponed to larger values of this parameter when $R e$ is decreased keeping the other parameters in (2.7) constant. These results can be understood noticing that the effect of the inertia of the liquid (left-hand side of (2.5)) decreases with $R e$ in a leading region of the jet which may include the current transfer region. Only the axial viscous force (first term on the right-hand side of (2.5)) is left in this leading region to oppose the electric force that stretches the jet and, as a consequence, the radius of the jet decreases rapidly with streamwise distance until the effect of the inertia comes into play. (See Higuera 2010 for order-of-magnitude estimates in the absence of fluid inertia, which give $r_{s_{c t}} \sim a C a^{1 / 2} \Lambda^{-1 / 4} V_{\infty}^{-1 / 2}, x_{c t} \sim a C a V_{\infty}^{-2}$ and $I \sim I_{0} \Lambda^{1 / 2} V_{\infty}^{2}$ instead of (3.3).) This means that the velocity of the liquid increases when $R e$ decreases at constant $C a$, and so does the convection current $I_{s}=2 \pi r_{s} \sigma v$ since $2 \pi r_{s} \sigma \sim \epsilon_{0} A$ is independent of $R e$, as was mentioned above. The results (3.4) are still valid for very large values of $\mathrm{Ca}$.

The length of the relaxation region at the outlet of the tube where the density of surface charge rises towards its equilibrium value $\epsilon_{0} E_{1 n}$ (see (2.2)) is $\ell_{e} \sim v t_{e} \sim \epsilon_{0} \epsilon Q / K a^{2}=a \epsilon C a / \Lambda$, where $t_{e}=\epsilon_{0} \epsilon / K$ is the electric relaxation time of the liquid and $v \sim Q / a^{2}$ in the framework of the quasi-unidirectional approximation of $\$ 2$. The length of the current transfer region $x_{c t}$ in (3.4) ceases to be large compared to $\ell_{e}$ when $C a$ increases to values of order $\Lambda / \epsilon^{1 / 2}$, which are somewhat larger than in the experiments of Riboux et al. (2011). The free surface charge fails to screen the liquid in the relaxation region from the outer field for any value of $\mathrm{Ca}$, and it also fails to screen the liquid downstream of the relaxation region when $\mathrm{Ca} \gg \Lambda / \epsilon^{1 / 2}$. However, the charge due to the polarization of the surface suffices to screen the relaxation region when $C a$ is small compared to $\Lambda / \epsilon^{1 / 2}$ and to screen a region of characteristic length $a \epsilon^{1 / 2}$ when $C a$ is large compared to $\Lambda / \epsilon^{1 / 2}$; see Higuera (2008). The electric current ceases to increase linearly with $C a$ and tends to a limiting value of order $I_{0} \Lambda V_{\infty} / \epsilon^{1 / 2}$ under these conditions.

Far downstream in the jet most of the current is due to the convection of the surface charge, $I \approx I_{s}$, and the momentum balance (2.5) reduces to $\partial\left(\pi r_{s}^{2} \rho v^{2}\right) / \partial x \approx 2 \pi r_{s} \tau_{t}^{e}$. Since $\tau_{t}^{e} \approx \sigma A / x, 2 \pi r_{s} \sigma \approx I / v$ and $v=Q / \pi r_{s}^{2}$, this equation can be integrated to give

$$
\frac{r_{s}}{r_{s_{0}}}=\left[1+\frac{2 \pi^{2} I A r_{s_{0}}^{4}}{\rho Q^{3}} \ln \frac{x}{x_{0}}\right]^{-1 / 4},
$$

where $x_{0}=O\left(x_{c t}\right)$ and $r_{s_{0}}=r_{s}\left(x_{0}\right)=O\left(r_{s_{c t}}\right)$ is an integration constant. The factor $2 \pi^{2} I A r_{s_{0}}^{4} / \rho Q^{3}$ is of order unity when (3.3) is applicable, and small of $O\left(V_{\infty}^{2} / R e C a^{2}\right)$ when (3.4) is applicable, which means that the radius of the jet is effectively constant (at $r_{s_{0}} \approx a$ ) up to very large distances. The velocity of the liquid and the charge per unit length of the jet, $2 \pi r_{s} \sigma$, are also constant, so that the far jet plus its image in the metallic tube acts as a line of charge of uniform strength. This has the potential to introduce non-local effects which have no analogue for a jet in the electric field of 
a Taylor cone, for which the strength of the line of charge decreases as $x^{-1 / 4}$. Thus, while a uniform line of charge induces only a radial field which does not invalidate the estimates above for the current transfer region, this situation may change when the length of the tube or the interelectrode distance, or the distance at which the jet breaks into drops or undergoes whipping, are not very large compared to $x_{c t}$. In the numerical computations, truncating the jet at a certain distance $x_{\infty}$ from the tube amounts to adding a line of negative charge that extends from $x_{\infty}$ to infinity downstream. The axial field induced by this line of charge is of order $A / x_{\infty}$ in the current transfer region and adds to the real applied field. As a consequence, very large values of $x_{\infty}$, ranging from $200 a$ to $800 a$, have been needed to keep the effect of the truncation small.

\section{Conclusions}

Numerical computations of the flow and the electric field in the current transfer region of an electrified jet issuing from a long metallic tube kept at a high voltage relative to a far electrode have been carried out using a quasi-unidirectional approximation for the flow in the jet. The electric current computed as a function of the flow rate injected through the tube approaches a square root law for small flow rates, levels to a nearly uniform plateau at intermediate flow rates and tends to increase linearly at large flow rates. The current increases nearly linearly with the voltage applied to the metallic tube.

The radius of the jet increases with the flow rate and decreases when the applied voltage increases, due to the increase of the electric shear stress that stretches the jet. The stretching of the jet is moderate in the conditions of the computations. If this stretching is fully neglected, the density of surface charge is proportional to the applied voltage beyond a charge relaxation length at the beginning of the jet, and the velocity of the liquid is proportional to the flow rate, leading to a convection current proportional to the product of these two variables.

The length of the current transfer region first increases with the flow rate, extending into the region where the electric field decreases as the inverse of the distance to the tube, and then decreases, reflecting the fact that, when the flow rate is large, the convection current rises rapidly at the beginning of the jet and the surface charge needs not screen a long stretch of the jet from the applied field. However, the conduction current remaining in the jet decreases slowly with streamwise distance in the absence of noticeable stretching, and its contribution to the total current is sensitive to the conditions of the jet far downstream. The length of the current transfer region increases with the conductivity of the liquid and decreases when the applied voltage increases, at least for large values of this variable and moderate flow rates.

I am grateful to Professor I. G. Loscertales (Málaga) for many insightful comments. This work was supported by the Spanish MICINN through Project DPI2010-20450C03-01.

\section{REFERENCES}

Ashley, H. \& Landahl, M. 1965 Aerodynamics of Wings and Bodies, Chap. 6. Addison-Wesley.

Barrero, A. \& Loscertales, I. G. 2007 Micro- and nano-particles via capillary flows. Annu. Rev. Fluid Mech. 39, 89-106.

Cloupeau, M. \& Prunet-Foch, B. 1989 Electrostatic spraying of liquid in cone-jet mode. J. Electrostat. 22, 135-159. 
FENG, J. J. 2002 The stretching of an electrified non-Newtonian jet: a model for electrospinning. Phys. Fluids 14, 3912-3926.

Fernández de la Mora, J. 2007 The fluid dynamics of Taylor cones. Anmu. Rev. Fluid Mech. 39, $217-243$.

Fernández de la Mora, J. \& Loscertales, I. G. 1994 The current emitted by highly conducting Taylor cones. J. Fluid Mech. 260, 155-184.

Gañán-Calvo, A. M., Dávila, J. \& Barrero, A. 1997 Current and drop size in the electrospraying of liquids. Scaling laws. J. Aerosol Sci. 28, 249-275.

Gundabala, V. R., Vilanova, N. \& Fernández-Nieves, A. 2010 Current-voltage characteristic of electrospray processes in microfluidics. Phys. Rev. Lett. 105, 154503.

Hartman, R. P. A., Brunner, D. J., Camelot, D. M. A., Maridnissen, J. C. M. \& Scarlett, B. 1999 Electrohydrodynamic atomization in the cone-jet mode. Physical modeling of the liquid cone and jet. J. Aerosol Sci. 30, 823-849.

Higuera, F. J. 2003 Flow rate and electric current emitted by a Taylor cone. J. Fluid Mech. 484, 303-327.

Higuera, F. J. 2004 Current/flow rate characteristic of an electrospray with a small meniscus. J. Fluid Mech. 513, 239-246.

Higuera, F. J. 2008 Breakup of a supported drop of a viscous conducting liquid in a uniform electric field. Phys. Rev. E 78, 016314.

Higuera, F. J. 2010 Electrodispersion of a liquid of finite electrical conductivity in an immiscible dielectric liquid. Phys. Fluids 22, 112107.

Hohman, M. M., Shin, M., Rutledge, G. \& Brenner M. P. 2001 Electrospinning and electrically forced jets. II. Applications. Phys. Fluids 13, 2221-2236.

Landau, L. D. \& Lifshitz, E. M. 1960 Electrodynamics of Continuous Media. Pergamon.

Larriba, C. \& Fernández de la Mora, J. 2010 Electrospraying insulating liquids via charged nanodrops injection from the Taylor cone of an ionic liquid. Phys. Fluids 22, 072002.

Riboux, G., Marín, A. G., Loscertales, I. G. \& Barrero, A. 2011 Whipping instability characterization of an electrified visco-capillary jet. J. Fluid Mech. 671, 226-253.

SAville, D. A. 1997 Electrohydrodynamics: the Taylor-Melcher leaky dielectric model. Annu. Rev. Fluid Mech. 29, 27-64.

Shin, Y. M., Hohman, M. M., Brenner, M. P. \& Rutledge, G. C. 2001 Experimental characterization of electrospinning: the electrically forced jet and instabilities. Polymer 42, 9955-9967.

TAYLOR, G. I. 1964 Disintegration of water drops in an electric field. Proc. R. Soc. Lond. A 280, $383-397$. 